
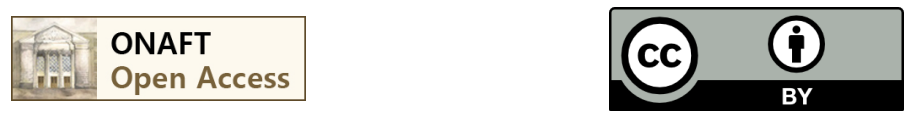

\title{
K-theory and phase transitions at high energies
}

\section{T.V. Obikhod}

\begin{abstract}
The duality between $E_{8} \times E_{8}$ heteritic string on manifold $K 3 \times T^{2}$ and Type IIA string compactified on a Calabi-Yau manifold induces a correspondence between vector bundles on $K 3 \times T^{2}$ and Calabi-Yau manifolds. Vector bundles over compact base space $K 3 \times T^{2}$ form the set of isomorphism classes, which is a semi-ring under the operation of Whitney sum and tensor product. The construction of semi-ring $V$ ect $X$ of isomorphism classes of complex vector bundles over $X$ leads to the ring $K X=K(V$ ect $X)$, called Grothendieck group. As $K 3$ has no isometries and no non-trivial one-cycles, so vector bundle winding modes arise from the $T^{2}$ compactification. Since we have focused on supergravity in $d=11$, there exist solutions in $d=10$ for which space-time is Minkowski space and extra dimensions are $K 3 \times T^{2}$. The complete set of soliton solutions of supergravity theory is characterized by $R R$ charges, identified by K-theory. Toric presentation of Calabi-Yau through Batyrev's toric approximation enables us to connect transitions between Calabi-Yau manifolds, classified by enhanced symmetry group, with K-theory classification.
\end{abstract}

Keywords M-theory · vector bundles · K-theory

UDK 539.12 


\section{Introduction}

M-theory, that first conjectured by Edward Witten in the spring of 1995, unifies all versions of string theory: type I, type IIA, type IIB, and two flavors of heterotic string theory $\left(\mathrm{SO}(32)\right.$ and $\left.E_{8} \times E_{8}\right)$. Each of these five string theories is limiting case of M-theory, and should be approximated by eleven-dimensional supergravity at low energies. M-theory is connected with the AdS/CFT correspondence and should describe branes [1]. For connection of M-theory with experimental data is used the mechanism of compactification of its extra dimensions to four-dimensional world, that can be verified at the LHC. One of the fundamental questions of theoretical high energy physics is the question of phase transitions of solitonic states, like D-branes. Due to the higher dimensional models, ADD or RS [2] in some scenarios involving extra dimensions of space, the Planck mass can be as low as the $\mathrm{TeV}$ range. As the Large Hadron Collider (LHC) has energy $14 \mathrm{TeV}$ for proton-proton collisions it was argued [3] that black hole production could be an important and observable effect at the LHC or future higher-energy colliders. Such quantum black holes or other high energetic silitonic objects should decay emitting sprays of particles that could be seen by detectors.

The purpose of the article is to study such solitonic objects with the help of effective model - D-branes. Our attempts for connection string theory with experiment are focused on the compactification of extra dimensions to $K 3 \times T^{2}$ for construction models of our four-dimensional world. The studied cases involve higher-dimensional spaces - D6-branes in for-dimensional space, for example. In section 2 we'll consider the equivalence of vector bundles on $K 3 \times T^{2}$ and CalabiYau manifolds. We are dealing with a special type of Calabi-Yau manifolds, when it is both an elliptic bundle over Hirzebruch surface $F_{2 k}$ and K3-fibration over the one dimensional projective space. In accordance with [4] there is the correspondence between the heterotic string compactifications on $K 3 \times T^{2}$ and type II string compactifications on Calabi-Yau threefolds of such type. This is connected with fact that for compactification of the heterotic string on $K 3 \times T^{2}$ is used the embedding, equating the spin connection of the manifold with the gauge connection. Through examples, it is possible that the moduli spaces of many different $\mathrm{N}=2$ heterotic vacua are connected in a similar way to type II string compactifications on Calabi-Yau. To each type of Calabi-Yau corresponds its enhanced symmetry according to Batyrev's toric approximation. In section 3 will be presented K-theory description of vector bundles over $K 3 \times T^{2}$. In section 4 we consider D-brane RR charge calculation corresponding to orbifold. 
The conclusion is connected with received fact that breaking of enhanced symmetries of Calabi-Yau to Standard model is connected with K-theory description of vector bundles over $K 3 \times T^{2}$, which differ from each other by the rank of the bundle.

\section{Calabi-Yau transitions}

From [5] we know that there exist duality between $(0,4)$ compactifications of the $E_{8} \times E_{8}$ heterotic string on the manifold $K 3 \times T^{2}$ and the type IIA string compactified on a Calabi-Yau manifold [6]. This duality corresponds to the equivalence of vector bundles on $K 3 \times T^{2}$ and Calabi-Yau manifolds. This Calabi-Yau are of special type - K3-fibration over $\mathrm{P}^{1}$ projective space. In the context of Mand F-theory [7] the dynamics on the moduli space of string theory would allow to determine the physical ground state of the string. The criterion which distinguishes between different vacua of string theory is the compactifications of these theories to three and four dimensions, in particular to CalabiYau fourfolds. Toric description of elliptic Calabi-Yau manifold according to Batyrev's approximation [8] can be realized by dual polyhedron which is devided by triangle on the top and bottom , For fourfolds of type $X_{18 k+18}(1,1,1,3 k, 6 k+6,9 k+9)$ the gauge groups are written in the following way [9]:

$$
\begin{gathered}
H \times S U(1) \quad \text { for } k=1, \\
H \times S O(8) \quad \text { for } k=2, \\
H \times E_{6} \quad \text { for } k=3, \\
H \times E_{7} \quad \text { for } k=4, \\
H \times E_{8} \quad \text { for } k=5, \\
H \times E_{8} \quad \text { for } k=6 .
\end{gathered}
$$

Thus, to each type of Calabi-Yau corresponds its enhanced symmetry. The moduli space of string theory vacuum can be deformed by vevs through breaking the gauge group. For example, $E_{8}$ can be completely broken through the chain

$$
E_{8} \rightarrow E_{7} \rightarrow E_{6} \rightarrow S O(10) \rightarrow S U(5) \rightarrow S U(4) \rightarrow S U(3) \rightarrow S U(2) \rightarrow S U(1) .
$$

So, the breaking of the gauge group is connected with phase transitions between different Calabi-Yau manifolds. 


\section{K-theory description of vector bundles over $K 3 \times T 2$}

As transitions between Calabi-Yau, described in such technique, are known, it would be interesting to understand an adequate mathematical method for description of transitions between different vector bundles over compact base space $K 3 \times T^{2}$ according to duality [5]. The studying of such vector bundles was performed by [9], where the bundle $\mathrm{V}$ on $K 3 \times T^{2}$ is fixed as follows

$$
V=\oplus_{i} p_{1}^{*} L_{x_{i}} \otimes p_{2}^{*} M_{i}
$$

where $L_{x_{i}}$ are the line bundles on $T^{2}$ corresponding to $x_{i}$ - a point in the dual torus $\check{T}^{2}, p_{1}$ and $p_{2}$ are projections from the product $K 3 \times T^{2}$ to the factors $T^{2}$ and $K 3$, respectively.

As is known from [11], vector bundles over compact base spaces form the set of isomorphism classes of vector bundles over $X$ (in our case $X$ is $K 3 \times T^{2}$ ). This is a semi-ring under the operations of Whitney sum and tensor product. It contains the disjoint union

$$
\operatorname{Vect} X=\bigcup_{d=0}^{\infty} \operatorname{Vect}_{d} X
$$

where $\operatorname{Vect}_{d} X$ comprises the classes of vector bundles of rank d. Such construction of semi-ring Vect $X$ of isomorphism classes of complex vector bundles over $X$ leads to the ring $K X:=K(\operatorname{Vect} X)$, which is called a Grothendieck group a contravariant functor from compact topological spaces to rings. From [12] is known that, there exists the isomorphism of free sheaves of rank $n$ and classes of vector bundles of rank $n$. The category $\operatorname{Vec}_{r}(X)$ of vector bundles of rank $\mathrm{r}$ on $X$ and the category $\operatorname{Loc}_{r}(X)$ of locally free sheaves of rank $\mathrm{r}$ on $X$ are equivalent, as defined by Hartshorne, [11]. In [13] is written about D-brane as locally free sheaf. From [14] it has been observed (for example, as in [15]) that branes supported on complex submanifolds of complex varieties are naturally described in terms of coherent sheaves. Therefore, Grothendieck groups of coherent sheaves, the holomorphic version of K-theory, can be used to describe D-branes in the case that all D-branes are wrapped on complex submanifolds.

\section{String theory and RR charge}

Since we are dealing with M-theory theory of five string theories, it is necessary to say that string looks like an ordinary particle, with its mass and charge. A physical object that generalizes the notion of a point particle to higher dimensions is a brane. A point particle can be viewed as a brane of dimension zero, 
while a string can be viewed as a brane of dimension one. It is also possible to consider higher-dimensional branes. In dimension p, they are called p-branes. Branes are dynamical objects which can propagate through spacetime according to the rules of quantum mechanics. They can have mass and other attributes such as RR-charge. Much of the current research in M-theory attempts to better understand the properties of these branes. So, the studying of D-brane classification with the help of K-theory description of RR-charge is of great importance.

Generally believed of K-theory as a "poor man's derived category" that knows only about D-brane charge [13]. A D-brane charge corresponding to a vector bundle $\mathrm{E}$ is given by formula

$$
Q(E)=\operatorname{ch}(E) \sqrt{t d(X)},
$$

where $\operatorname{ch}(E)$ is the Chern character of $E$ and $t d(X)$ is the Todd class of the tangent bundle of $X$. The charge of a D-brane given by a coherent sheaf can be computed using the Grothendieck-Riemann-Roch theorem [13].

As is stressed in [16] blowing-up of $T^{4} / Z_{2}$ is $\mathrm{K} 3$ space. Therefore, instead of $K 3 \times T^{2}$ space we can consider $T^{6} / Z_{2}$ space - orbifold. D-brane that passes through an orbifold fixed point carries RR charge. In a supergravity approximation we can take the large volume limit to describe the backgrounds and these are RR backgrounds [17]. Large volume charges are connected with RR charges by formula:

$$
Q_{4}=n_{1}-2 n_{2}+n_{3}, Q_{2}=-n_{1}+n_{2}, Q_{0}=\frac{n_{1}+n_{2}}{2}
$$

which define the Chern character

$$
\operatorname{ch}\left(n_{1} n_{2} n_{3}\right)=Q_{4}+Q_{2} \omega+Q_{0} \omega^{2},
$$

$\omega$ is Wu parameter. The rank of the vector bundle $E$ is $Q_{4}, Q_{2}=c_{1}, Q_{0}=\frac{c_{1}^{2}}{2}$, where $c_{1}$ is the first Chern class. From [10] the first Chern class of $S U(N)$ bundle $E$ over $K 3 \times T^{2}$, for example, is zero, therefore $Q_{2}=0$ and $Q_{0}=0$ and vector bundles over $K 3 \times T^{2}$ are differ through the rank of the bundle.

\section{Conclusion}

The conclusion is connected with the fact that morphisms of distinct CalabiYau permit an interpretation in terms of topological K theory or Grothendieck groups. In spite of the fact that $K 3 T^{4} / Z_{2}$ and the full space $K 3 \times T^{2} T^{6} / Z_{2}$, we can't use the notion of Aspinwall [13] that D-branes on the orbifold $C_{d} / G$ and open strings between them are described by the derived category of McKay 
quiver representations (with relations) because we cannot have the derived category of a "compact" CY manifold represented by a quiver. Anyway, in spite of the fact that K-theory contains much less information than the derived category this is the beginning for understanding of brane classification when the symmetry is broken from $\mathrm{E}_{8}$ to the Standard molel. According to [18] when we focus our attention on supergravity in $d=11$, solutions also exist for $N=1$ in $d=10$, 9 and 8 dimensions for which spacetime is Minkowski space and for which the extra dimensions are $K 3 \times T 2, K 3 \times S^{1}$ and $K 3$, respectively. Starting from $64+64$ components of $\mathrm{N}=1$ in $\mathrm{d}=10$, we obtain $96+96$ components in $\mathrm{d}=4$ of $\mathrm{N}=2$ supergravity and so on to the Standard model.

\section{References}

1. Duff, Michael; Howe, Paul; Inami, Takeo; Stelle, Kellogg: Superstrings in $D=10$ from supermembranes in $\mathrm{D}=11$. Nuclear Physics B191 (1), 70-74 (1987).

2. Arkani-Hamed N., Dimopoulos S., Dvali G.: The Hierarchy problem and new dimensions at a millimeter. Physics Letters B429 (3-4), 263-272 (1998), arXiv:hep-ph/9803315;

Arkani-Hamed N., Dimopoulos S., Dvali G.: Phenomenology, astrophysics and cosmology of theories with submillimeter dimensions and $\mathrm{TeV}$ scale quantum gravity. Physical Review D59 (8), 086004 (1999), arXiv:hep-ph/9807344;

Randall, L., Sundrum, R.: Large Mass Hierarchy from a Small Extra Dimension. Physical Review Letters 83 (17), 3370-3373 (1999), arXiv:hep-ph/9905221;

3. Giddings, S. B., Thomas, S. D.: High-energy colliders as black hole factories: The End of short distance physics. Phys. Rev. D65 (5), 056010 (2002), arXiv:hep-ph/0106219;

Dimopoulos, S., Landsberg, G. L.: Black Holes at the Large Hadron Collider. Phys. Rev. Lett. 87 (16), 161602 (2001), arXiv:hep-ph/0106295;

Johnson, George: Physicists Strive to Build A Black Hole. The New York Times. Retrieved 2010-05-12;

The case for mini black holes. CERN courier. November 2004.

4. Kachru Shamit, Vafa Cumrun: Exact Results for $\mathrm{N}=2$ Compactifications of Heterotic Strings. Nucl. Phys. B450, 69-89 (1995).

5. Candelas Philip; Font Anamaria: Duality Between the Webs of Heterotic and Type II Vacua, arXiv: hep-th/9603170.

6. Klemm A., Lerche W., and Mayr P.: K3-Fibrations and Heterotic-Type II String Duality. Phys.Lett. B357, 313-322 (1995), hep-th/9506112.

7. Witten E.: String Theory Dynamics in Various Dimensions. Nucl.Phys. B443, 85-126 (1995), hep-th/9503124;

Hull C. and Townsend P. K.: Enhanced Gauge Symmetries in Superstring Theory. Nucl.Phys. B451, 525-546 (1995), hep-th/9505073;

Schwarz J.: The power of M theory. Phys. Lett. B367, 97-103 (1996), hep-th/9510086;

Vafa C.: Evidence for F-theory. Nucl. Phys. B469, 403-418 (1996), hep-th/9602022;

Morrison D. R. and Vafa C.: Compactifications of F-theory on Calabi-Yau threefolds I,II, hep-th/9602114, hep-th/9603161. 
8. Batyrev V. V.: Variations of the Mixed Hodge Structure of Affine Hypersurfaces in Algebraic Tori. Duke Math. J. 69, 349-409 (1993).

9. Malyuta Yu. and Obikhod T.: Compactifications of F-Theory on Calabi-Yau Fourfolds, arXiv:hep-th/9803241.

10. Bershadsky Michael; Sadov Vladimir: F Theory on K3xK3 and Instantons on 7-branes. Nucl.Phys. B510, 232-246 (1998).

11. K. Wirthmüller: Vector Bundles and K-Theory 2011/2012.

12. Hartshorne, Robin: Algebraic Geometry.- Berlin, New York: Springer-Verlag, (1977), 496p.

13. Aspinwall Paul S.: D-Branes on Calabi-Yau Manifolds, arXiv:hep-th/0403166.

14. Sharpe Eric R.: D-Branes, Derived Categories, and Grothendieck Groups. Nucl.Phys. B561, 433-450 (1999).

15. Harvey J. and Moore G.: On the algebras of BPS states. Comm. Math. Phys. 197, 489-519 (1998), hep-th/9609017;

Hori K. and $\mathrm{Oz}$ Y.: F-theory, T-duality on K3 surfaces and $\mathrm{N}=2$ supersymmetric gauge theories in four dimensions. Nucl. Phys. B501, 97-108 (1997), hep-th/9702173.

16. Braun A.: Elliptic K3s, T 4/Z2 and Enriques involutions. Bonn - May 19th, 2009, http://hep.itp.tuwien.ac.at/ abraun/bonn.pdf.

17. Becker Katrin: Torsional heterotic geometries. "14th Itzykson Meeting" IPHT, Saclay, June 19, 2009.

18. The World in Eleven Dimensions: Supergravity, Supermembranes and M-theory. Edited by. M.J. Duff. University of Michigan, (1999), 513p.

\section{T.V. Obikhod}

